commonly regarded as the pathognomonic signs of wound of the lung were at first present, except escape of air (traumatopnoea) from the wound of exit and pain in the chest at the seat of the injuries. The value of the conservative treatment of gunshot wounds of joints is worthy of notice, together with the success of the dry method of treating wounds antiseptically.

Dusa.

\section{SOME OF THE MOST FREQUENT CAUSES OF SUDDEN DEATH.}

By FRANCIS EWENS, M.D., D.P.H.,

IATT hOUSE PHYSICIAN, KING'S COLLEGE HOSPITAL, AND IRESIDENT MEDICAL OFFICER TO THE PUBLIC DISPENSARY.

EXcrudiNG those cases of death from the various kinds of suicide, violence, or accident, there is a large number (between 3000 and 4000 annually) in which that event occurs quite suddenly, and though in the majority some illness is obviously present at the time, still in many also that condition may be ill-defined, latent, or quite unsuspected. The details of such cases are, at least, of great interest, and may possibly throw some additional light on the direct mechanism of death at all times. The subject bas, however, been so exhaustively treated in Dr. Ogston's paper, ${ }^{1}$ by Dr. West in his paper, ${ }^{2}$ and other writers, and such full lists of causes are given in all the principal text-books, that I feel some apology is necessary before venturing to put forward even the brief résume of the details from all their sources, together with a few additional cases scattered through the various periodicals and journals, and one or two which happen to have come under my own notice. Before doing so, however, it is necessary to remark that the cases occurring among young children require to be separated from those seen in adults, their cause being totally different. Among children, as Dr. West has shown, the most frequent cause of sudden death is some form of laryngeal obstruction, generally laryngismus stridulus; another equally frequent being the acute stage of pneumonia, in which they sometimes die suddenly, even with the disease unsuspected, these, as Ogston has shown, forming a large proportion of the cases of death attributed to having been overlain. Another cause is incomplete expansion of the lung, children in this condition often dying suddenly a week or so after birth. A smaller number die of pulmonary apoplexy, and a still smaller of exbaustion during chronic diarrhcea \&c.

Among adults the chief causes in their order of freinency are as follows :-

1. Large blood effusion into the brain or nembranes, causing a rapidly fatal coma, those into the medulla being most likely to do so, death in such cases sometimes occurring almost instantaneously.

2. Diseases of the heart, bloodvessels, \&c. (a) Fatty degeneration. (b) Valvular diseases. These are generally cases of aortic incompetence, and next most frequently mitral stenosis. (c) Cases in which death occurs during an attack of angina pectoris. In these cases the heart is generally found to be fatty and with its coronary arteries atheromatous, or having the same change in the aortic walls just outside the semilunar valves. In rarer cases a small aneurysm may be found. The most common lesion in fatal cases seems to be a coronary artery obstructed with atheroma. (d) Ruptured heart. The rupture is usually on the left ventricle in a heart affected with fatty degeneration. (e) Some rare cases of interstitial abscess and of pericarditis. $(f)$ Rupture of an abdominal, thoracic, or intracranial aneurysm. $(g)$ Pulmonary thrombosis or embolism. Death from these causes occurs much oftener than is generally supposed, forming by far the largest proportion of those cases of sudden death happening some days after delivery, pulmonary thrombosis being then much more common than embolism. 'The symptoms of the affection are fairly distinctive and well worthy of notice. An intense attack of dyspnoea comes on suddenly, the patient rapidly becoming cyanosed, with swollen cervical veins. The heart acts tumultuously and rapidly; a basic bruit can be often heard to the left of the sternum, and the pulse becomes feeble and thread-like, there being all the Medical Times, 1859 . time, as can be heard on auscultation, no obstruction to the air entering the lungs. Tessillon ${ }^{3}$ gives a distinction between an attack of this kind and one of pulmonary embolismthat in the latter the lips and face become very pallid, are not cyanosed, and that there is less respiratory anxiey and dyspnoca, death in the latter seeming to be due more so shock thon asphyxia. (h) Impaction of a detached clot (generally coming from the dilated auricular appendix) ir a contracted mitral orifice is sometimes seen, and when it occurs causes very rapid death, preceded by a sudden faint, an imperceptible pulse, but a strongly acting heart, gaspizg respiration, cyanosis, and insensibility. A case of this kind occurred at King's College Hospital some three years ago. A woman, admitted under the care of Dr. Yeo, with mitral stenosis and gangrene of the forearm from embolism, sut. denly died with exactly these symptoms, and another case is recorded by $\mathrm{Van}$ de $\mathrm{By}^{4}{ }^{4}$ as ending in this way in a patient with mitral regurgitation. (i) Cases of capillary emboli are quoted in which death occurred very quickly, but they were probably cases of ulcerative endocarditis. (j) Rupture of the coronary artery. An interesting case of death from this cause is recorded in THE LANCET of April 20th of this year.

3. Affections of the lungs and pleura. (a) Pneumonia: Death sometimes occurs very suddenly in this disease; it usually does so during the stage of red hepatisation, being then due to a clot in the pulmonary artery and the liability to it being out of all proportion to the severity of the symptoms or the amount of lung substance involved. The following case well exemplifies these particulars. A patient of my own was suffering from pneumonia of the right lung, the middle lobe being alone involved. On the third day she felt so little ill that she persisted in getting up to dress, and got out of bed for that purpose, walked across the room, and fell down dead. (b) Pulmonary apoplexy and hæmato-thorax sometimes cause sudden death. $(c)$ In young children rapid effusion of fluid into the pleuræ has been known to cause sudden death, but there seems no recorded instance of it having done so in the adult.

4 Certain abdominal diseases. (a) Rupture of a gastric ulcer. These cases are peculiar, in that sometimes death does not occur immediately after rupture, but suddenly some days even afterwards, without any fresh symptom or post-mortem signs to account for it. A case of this nature occurred in King's College Hospital in 1887, when a young girl came in under Dr. Duffin's care, with an obscure history of a sudden attack of abdominal pain a week previously and subsequent abdominal tenderness, and vomiting and constipation. She woke up the second night complaining of an attack of pain, and, while speaking, turned pale and died almost instanteously. Post-mortem examination showed a tiny ulcer in the anterior wall of the stomach, with a pinhole rupture and lymph around it for a inch or so, but nothing fresh to account for the sudden termination. (b) Sudden history of visceral abscesses and of extra-uterine fotation or of a hæmatocele. (c) Ruptured uterus. $(d)$ Volvulus.

5. Cholera sometimes kills quickly, and there are several other diseases in which death occurs suddenly, often enough to be noticeable-namely, $(a)$ diphtheria. In this affection the pulse sometimes becomes much slowed, and fatal syncope may happen even after all symptoms of the throat affection have subsided some time. (b) Typhoid. Dieulafoy quotes fifty cases wheresudden death occurred. This happened in nearly all from the seventeenth to the nineteentil day of illness. He puts forward the theory that the fatal result is due either to change in the heart muscle or a reflex shock from the bowel to the medulla, and cites other cases where reflex irritation of the intestine caused death, post mortem a foreign body being found in the bowel. (c) Acute peritonitis. (d) Some zymotic diseases.

6. Cerebral tumour. This affection sometimes ends fatally with great suddenness, and in such case there is often no appreciable fresh change to account for the event to be found post mortem. Two cases exemplifying this will be alluded to later.

7. Labour is sometimes attendant or followed by sudden death, due to $(a)$ pulmonary thrombosis or embolism (these cases happening some days after as a rule), (b) ruptured uterus, $(c)$ air in the uterine sinuses, $(d)$ fatal syncope, (e) rupture of a varicocele of ovarian veins, the cases recorded being about all on the left side. 
8. Sunstroke may be attended with a rapidly fatal result. 9. Some few rarer causes remain: $(a)$ The swallowing large dranghts of cold water when heated. (b) Mental emotions. These two causes are mentioned in all the textbooks, but I have not been able to find detailed accounts of their occurrence anywhere. (c) Foreign bodies accidentally swallowed, obstructing the glottis, and causing fatal shock. As to the exact manner in which these canses act in producing sudden death little can be said; it is, however, but reasonable to suppose that the suddenness of its occurrence is a result of the unimpeded, or at any rate very preponderating, action of the mechanism by which life is ended in all cases, and that an investigation into the exact manner in which these particular cases are brought about would throw much light on that of death in general. However, even the scanty facts at present known of these cases throw some little doubt on the accuracy of the generally accepted division of the causes of death into those due to stoppage of the circulation or of the respiration, Bichat's third cause- "death beginning at the head"-being now omitted by many writers. It is certain that the effect of irritation of the vagus on the human being is similar to the results obtained in experiments on animals, in that it will produce slowing of the heart's beats, and, if persisted in, death. Without referring to the well-known case of Czermak, who experimented on himself in this way, or to those of death in patients affected with aneurysm not far ad. vanced enough to have produced that result alone, and where the vagus has been found to be stretched and pressed upon by the swelling, a very interesting case is quoted in the Medical Times and Gazette, June, 1884, in which a child aged three died very suddenly, and where the apparent cause of death was found to be an inflamed vagus, pressed upon as it lay between a caseous bronchial gland and an abscess sac. Another case is quoted in THE LANCET of January, 1880, in which death resulted suddenly from dilated rentricle pressing upon a closed fourth ventricle and the vogus centre. It is probable that those cases following diphtheria where death occurs suddenly, often preceded by an extremely slow pulse, which latter symptom without a fatal result is seen, are produced by disease of the vagus. Again, although it may be difficult or impossible experimentally to injure the respiratory centre in animals without also affecting the cardiac centre and so causing death, it is not so in disease in man, as cases of sudden stoppage of the respiration, while the pulse still continues to beat until the patients die asphyxiated are seen in some cases of death from cerebral tumour. Two instances of this happened to come under my own notice : 1. A man in King's College Hospital in 1887, under the care of Dr. Ferrier, with a tumour in the frontal region, was in his usual chronic condition, as he had remained some months; his respiration suddenly quite ceased, the heart going on beating apparently as usual ; artificial respiration was kept up for over an hour without the slightest return of normal respiration, and he died at the end of that time. 2. Another similar case was that of a woman with an abscess in the temporo-sphenoidal lobe, and in whom respiration also suddenly ceased while the heart continued to beat. It is worthy of note that absolutely nothing was found post mortem to account for the sudden termination of her illness. Of the opposite condition--continuance of respiration without heart beats-there does not seem to be any recorded instances. Again, the suddenness of the death is in direct ratio with that of the stoppage of the heart, and of all causes those acting directly by the nervous system-i.e., through the vagi either from the medulla or by damage of that nerve-seem to act quickest ; as also those cases where it is so brought about by what is generally termed "reflex shock," as in intestinal cases \&c., and those of sudden stoppage of the blood-supply to the brain, as in ligature of arteries, which may be both supposed to act in the same way. Also sudden death is slowest brought about when produced by the circulation of non-oxygenated blood, and in this particular it is worthy of note that of the preceding list of causes but very few-such as the comas of cerebral hæmorrhage, pulmonary thrombosis, and apoplexygive the post-mortem or clinical evidences of death from asphyxia; and that in all these death is slower (except, perhaps, pulmonary thrombosis) than in most of the other affections, in some of which it is often absolutely sudden.

Therefore, having regard to the fact that stoppage of the respiration is not in itself fatal until the heart stops; that stoppage of the heart's uction seems invariably and immediately to be so; that stoppage of the heart may be caused by nervous influence alone, when all the other organs seem apparently healthy, as in the case of death from cerebral tumour, vagus pressure, \&c., above alluded to, as well as cases of death from lightning and reflex shock and that it is probable that the very" "cardiac cases" themselves may be explained by anomia of the cardiac plexuses,-it is therefore possible, and a little probable, that the immediate cause of death at all times is a nervous influence stopping the heart; that "cerebral " death is the only form, that from other causes, as from aspliyxia, being a misnomer, that condition only being fatal when the non-oxygenation of the blood is sufficiently intense to excite the medullary cardiac centre.

Highgate, $\mathbf{N}$.

\section{ON THE VALUE OF EMACIATION.}

\section{BY J. B. NIAS, M.B. OxoN., M.R.C.P. LoND.}

EMACIATION is a prominent feature in many diseases. Many of the phenomena of disease are, in reality, efforts at repair. It will, therefore, be advantageous to inquire if some good purpose is not served by emaciation. To begin with diseases which affect the circulation. In many such we may note that the patient will rapidly lose flesh, and that when the loss has proceeded to a certain degree, it is arrested. The patient becomes and continues thin. Not only is it difficult to fatten him, but he is not much benefited by the attempt. Should his disease be arrested, but leave some injury behind, the patient will probably continue thin. Instinctively, in consequence, we look for the presence of disease, active or quiescent, in sparely developed persons. And, practically, we look for it in the territory of the circulation, either pulmonary or systemic, and generally find something. Such emaciation, ceasing at a certain point, does not much exceed what is seen in athletic training, and may be defined as an involuntary training, forced upon the patient by his ailment. What, briefly, is the benefit of training? Unusual effort in untrained men or animals is checked, not by muscular exhaustion, but by congestion of the pulmonary circulation and paralysis of the right ventricle of the heart. The effect of training is to enable the pulmonary circulation to keep pace with the increased activity of the systemic. Several changes concur to this end : dilatation of the lung and of its bloodvessels; hypertrophy of the right side of the heart; and most especially absorption of every tissue which is found to be superfluous for the effort in view. This absorption reduces the area of the systemic circulalation; and it is followed by a reduction in the quantity of blood, because a smaller quantity will suffice for active circulation through the leaser area. The individual so trained is in the condition of having a pulmonary territory larger than is necessary for his state of rest; and, consequently, with a greater margin for relief upon exertion. Conversely, the contrary condition of obesity develops the systemic circulation to the full capacity of the lungs, so that the least exertion will produce dyspnoea. Acute disease being like exertion, we can see the advantage of entering upon it in a state of training. The exhaustion to be feared is that of the heart's right ventricle; and the inconvenience of existing stimulants is, that they do not stimulate the right ventricle by itself.

To return to emaciation, as produced by disease. Let us first take the case of diseases of the lung. Should the territory of the pulmonary circulation be diminished by such a disease as phthisis, it would be impossible for the patient, without emaciation, to have an active circulation in the systemic area without danger of congestion of the lung. The feeble attempts that are made at hypertrophy of the lung in this disease are thus met half way. The same thing is true of all emphysema of the lungs, whether senile or morbid. Then we may consider the disorders of the greater circulation. In disease of the valves, when the muscle has to do the work of the valves, and in decay of the aorta, when the ventricle has to combine the aorta's duty with its own, the smaller the volume of blood to be dealt with the better. Without reduction of the area through which it flows, a reduced amount of blood would only result in a sluggish circulation. Emaciation in such cases is therefore salutary. Moreover, the capillaries are 